\title{
MODELO DE PLANIFICACIÓN PARA ACTUAR ANTE LA PANDEMIA
}

\author{
Crnl. de E.M.C. Carlos M. Cano Serrano ${ }^{1}$
}

\begin{abstract}
RESUMEN
La emergencia declarada por la OMS el 12 de marzo de 2020 desata en el mundo entero un escenario sin precedentes que aumenta y se potencializa aún más en un entorno plagado de incertidumbres, donde resulta difícil para los Estados y sus Fuerzas Armadas tratar de darle una "valoración" a la amenaza que se presente con la dinámica necesaria. Tanto en el Ecuador como en el resto del mundo, la rapidez en la evolución de los hechos, a escala nacional e internacional, requiere la adopción de medidas inmediatas y eficaces para hacer frente a esta coyuntura. Las circunstancias extraordinarias que concurren constituyen, sin duda, una crisis sanitaria sin precedentes y de enorme magnitud, tanto por el muy elevado número de ciudadanos afectados diariamente, como por el riesgo que atraviesa la supervivencia del Estado. Para ello, las FF.AA. han planteado un diseño operacional basado en la evolución y comportamiento de la enfermedad para entender el problema desde el ámbito operacional y de la seguridad nacional y con ello, plantear las diferentes estrategias y acciones que apoyen en la solución del problema, pasando de la formalidad de la planificación militar deliberada a la planificación en crisis, a través de la experiencia vivida entre los meses de marzo a julio del 2020. El presente artículo busca explorar nuevos modelos y diseños operacionales empleados en la dinámica de la búsqueda de la solución a este acontecimiento mundial; así como la aplicabilidad del modelo operacional empleado y la definición de su validez para las Operaciones de Apoyo a la Secretaría Nacional de Gestión de Riesgos, en respuesta a una crisis atípica como la presentada con la pandemia del Covid-19.
\end{abstract}

Palabras clave: Dinámica-Amenaza-Riesgo, Diseño-Operacional, Modelo-operacional, COVID-19, OperacionesApoyo, Gestión-de-riesgos.

\begin{abstract}
The emergency declared by the World Health Organization on March 12, 2020; unleashes an unprecedented scenario throughout the world that increases and becomes even more potent in an environment plagued with uncertainties, where it is difficult for States and their Armed Forces to try to give an "assessment" to the threat that arises with the dynamics necessary. Both in Ecuador and in the rest of the world, the rapidity in the evolution of events, on a national and international scale, requires the adoption of immediate and effective measures to face this situation. The extraordinary circumstances that occur constitute, without a doubt, an unprecedented health crisis of enormous magnitude, both due to the very high number of citizens affected daily and because of the risk that the State's survival faces. For this, the Ecuadorian Armed Forces have proposed an operational design based on the evolution and behavior of the disease, to understand the problem from the operational and national security scope and with it, propose the different strategies and actions that support the solution of the problem, going from the formality of deliberate military planning to planning in crisis, through experience lived between the months of March to July 2020. This article seeks to explore new models and operational designs used in the search for the solution to this world event; as well as the applicability of the operational model used and the definition of its validity for Support Operations to the National Secretariat for Risk Management in response to an atypical crisis such as the one presented with the COVID-19 pandemic.
\end{abstract}

Key words: Dynamic-Threat-Risk, Design-Operational, Model-operational, COVID-19, Operations-Support, Risk-management.

\footnotetext{
${ }^{1}$ cmcanos@ejercito.mil.ec

Agregado militar del Ecuador ante la República de Colombia

EJÉRCITO ECUATORIANO
} 


\section{Introducción}

T a Organización Mundial de la Salud elevó el 11 Lde marzo de 2020 la situación de emergencia de salud pública ocasionada por el COVID-19 a pandemia internacional, la rapidez en la evolución de los hechos, a escala nacional e internacional, requería la adopción de medidas inmediatas y eficaces para hacer frente a esta coyuntura en donde las circunstancias extraordinarias concurrieron en una crisis sanitaria sin precedentes y de enorme magnitud tanto por el muy elevado número de ciudadanos afectados diariamente por la enfermedad, como por el alto riesgo de que un "descontrol" de la situación amenace la supervivencia del Estado. Para entender mejor el "ambiente operacional" que se presenta, a continuación se presenta la presente línea de tiempo que describe el avance de la crisis epidemiológica en su primera etapa:

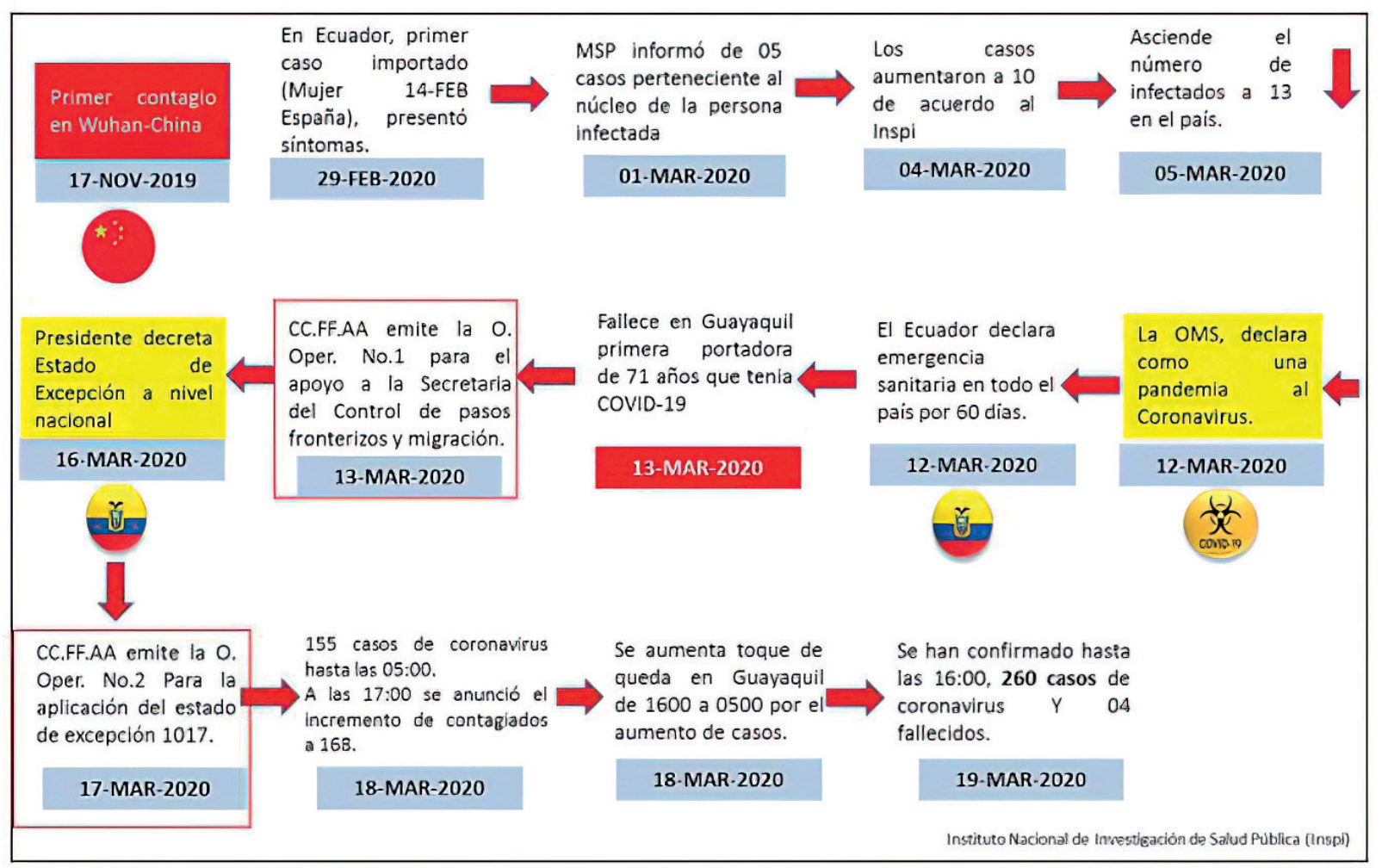

Figura 1: Línea de Tiempo de la Crisis, en su primera etapa ${ }^{1}$

En respuesta a este escenario, cuya compresión era bastante difusa, el 11 de marzo del 2020, el CC.FF. AA. realiza el su Plan de Contingencia "inicial" para Enfrentar la Pandemia del Coronavirus; y en base a ello y a la declaratoria de "pandemia mundial decretada por la OMS, dos días después, emite su 1era. Orden de Operaciones al mencionado plan, cuyo propósito principal era: "Apoyar al Ministerio de Salud Pública y al Servicio de Control Migratorio; realizando operaciones de seguridad en apoyo al control de los pasos fronterizos y puntos de ingresos al pais, apoyo logístico en el transporte de kit sanitarios y alimenticios y otras requeridas con orden".

Esta concepción inicial y esta tarea duraron apenas 4 días, ya que luego de la emisión del Decreto de Estado de Excepción 1017, el 17 de marzo de 2020, se emite la Orden de Operaciones No. 02 al mismo Plan de Contingencia, esta vez en apoyo a la Policía Nacional; y cuyo propósito principal fue: "el apoyar en el control de

${ }^{1}$ COVID 19 Cronología de una Crisis, pág. 33 la restricción vehicular y peatonal durante la emergencia del Covid-19 en el Ecuador", con especial atención a las provincias de Los Ríos, Guayas y Pichincha"; este hecho marcó el inicio real de las operaciones, sin embargo, el ambiente real operacional que se iba a vivir estaba muy lejos de comprenderse.

\section{Antecedentes}

Para estar en condiciones de "desarrollar la situación" y perfeccionar el plan inicial, el Comando Conjunto de las Fuerzas Armadas ecuatorianas, se planteó un diseño operacional basado en la evolución y comportamiento de la enfermedad para enfrentar el problema sanitario y de seguridad que se venía en nuestro país y con ello, plantear las diferentes acciones que apoyen en la solución de la crisis.

Se consideró, para el desarrollo inicial de la hipótesis, las siguientes "variables de partida":

En primer lugar, la estadística general de la Organización Mundial de la Salud (OMS), que establece que: "por cada persona contagiada se afectan tres". 
En segundo lugar, los siguientes datos:

- $80 \%$ de la población contagiada corresponde a diagnósticos leves y moderados.

- $\quad 15 \%$ de la población contagiada corresponde a diagnósticos de graves.

- $5 \%$ de la población contagiada corresponde a diagnóstico crítico.
- Existían a ese momento "353 camas en la Unidad de Cuidados Intensivos (UCI) del total de 3017 en 27 hospitales".

En tercer lugar, el caso cero (0) inició el 28 de febrero y para el 17 de marzo se registraban 199 contagios confirmados; con esos datos iniciales se pudo inferir la primera proyección lineal:

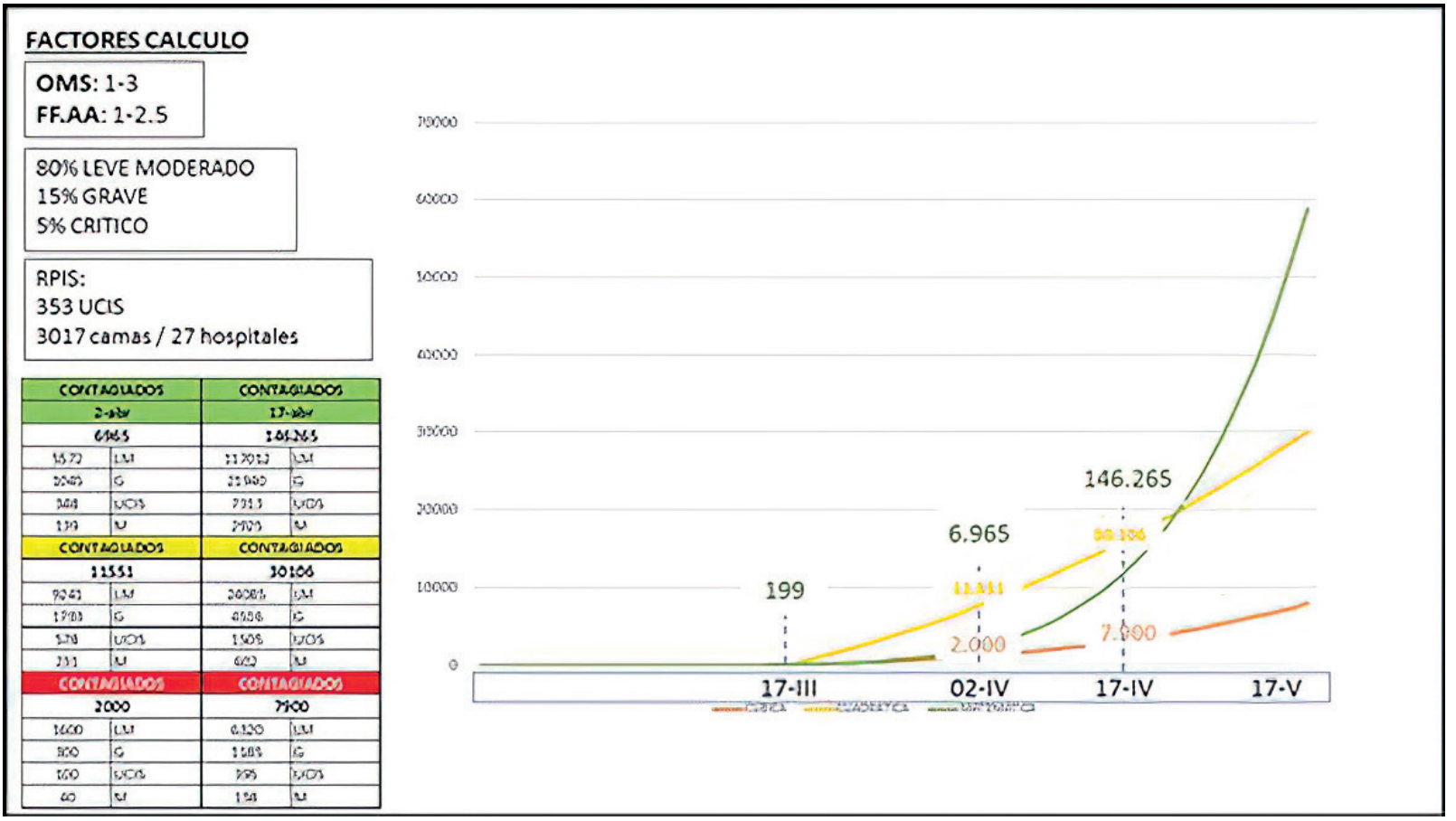

Figura 2: Proyección de casos de contagio en la población a partir de 19-MAR-020

Dando seguimiento al modelo lineal inicial presentado, se pudo inferir que:

1) Para el 2 de abril existirían 6965 contagiados, de los cuales 5572 se diagnostican como casos leves y moderados; 1045 como graves; 348 como críticos; 139 muertos y que corresponden a la primera cuarentena de 14 días considerada según el comportamiento del Covid-19. Con estos datos se llegaría a colapsar la disponibilidad de medios declarada por la Red Pública Integrada de Salud.

2) Del 2 de abril al $\mathbf{1 7}$ de abril, que corresponden a la segunda cuarentena de 14 días considerada según el comportamiento del Covid-19, se presentarían 146 265 contagiados, de los cuales 117.012 se diagnostican como casos leves y moderados; 21.939 como graves; 7313 como críticos; 2925 muertos.

Lo que nos permite visualizar en las siguientes proyecciones, 3 escenarios:

- Optimista: (color ROJO: producto de una regresión "cúbica")

- Tendencial: (color AMARILLO: producto de una regresión "cuadrática")

- Pesimista: (color VERDE: producto de una regresión "lineal")

Sin duda existieron y se presentaron a lo largo de la fase inicial de la crisis, otros modelos utilizados por distintas entidades a nivel nacional y mundial que pueden utilizar algoritmos más avanzados; más para fines del planeamiento y faseamiento de las operaciones el equipo mantuvo la hipótesis de un modelo lineal y basados en ese mismo algoritmo, se construyó un modelo de evolución de lo que representaría el riesgo de contagio dentro de los miembros de las FF.AA., ya que una de las principales preocupaciones operacionales iniciales, siempre fue: ¿hasta qué punto el riesgo de contagio en los miembros de las FF.AA. se constituiría realmente en una importante vulnerabilidad, que podría disminuir drásticamente las capacidades de las unidades del CC.FF.AA.?, escenario que lo que ventajosamente no sucedió, pero que debía preverse en la planificación y conducción de las operaciones plasmadas en la Figura 3 de la página siguiente. 


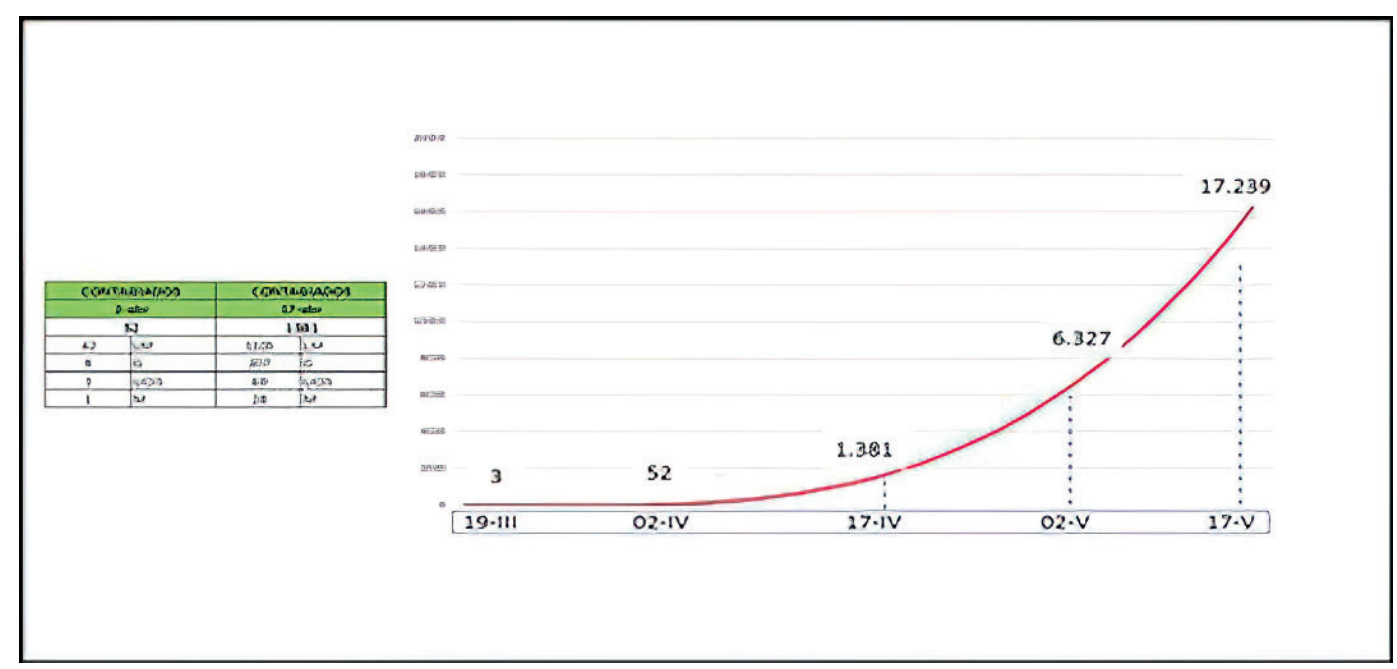

Figura 3: Proyección de casos de contagio en el personal de las FF. AA.

\section{Desarrollo}

En la realidad, a la primera quincena de marzo podían existir muchas ideas y pudo delinearse un plan inicial, pero realmente ninguno de nosotros podía decir a ciencia cierta que sabía lo que estaba por venir; por ello, aplicando los principios de la planificación militar, comenzamos en volcar todo nuestro esfuerzo inicial en la compresión del ambiente operacional, para ello se integró al equipo planificador a un epidemiólogo, contábamos con la asesoría telemática de dos doctores en el campo de la estadística y la matemática que mantenían actualizado un modelo mucho más completo que el nuestro, otra parte de nuestro equipo revisaba la doctrina americana, brasilera y la aún novedosa doctrina Damasco colombiana, buscando inferir el mejor camino o tratando de adaptar las operaciones NBQ a lo que estaba pasando. Es así que en ese proceso fuimos construyendo nuestro propio pensamiento y adaptando a "nuestra realidad" el problema que se planteaba.

Los datos presentados e inferidos en el modelo inicial, sirvieron para diseñar un modelo estratégico para la toma de decisiones, en donde se podía establecer las "fases y la tareas" que se debían cumplir en cada fase, con ello podíamos estructurar "un esquema operacional" que nos de luces de las misiones tipo tareas que debíamos entregar en ese momento a los Comandos Operacionales y las previsiones que debíamos tomar para cuando nos toque enfrentar las fases subsiguientes; basados en la premisa que "lo más importante será continuar con las medidas restrictivas que se señalan en el estado de excepción y progresivamente, hasta el 2 de abril, incrementar dichas medidas conforme avanza la pandemia, con el objetivo de no permitir que el número de contagios llegue al punto de inflexión en donde se salga del control la pandemia y se encuentre en grave riesgo la seguridad y supervivencia del Estado"; producto del análisis del diseño inicial del modelo, permitió inferir que el tiempo a considerar que duraría la operación sería aproximadamente entre dos meses y medio a tres.

\section{Modelo operacional de la crisis}

Con los datos presentados, el CC.FF.AA. desarrolla un modelo de conducción de la crisis en la que se visualizan 4 fases definidas:

(1) Una lera. fase de "Configuración inicial", desde el inicio de la crisis hasta antes del 17 de marzo de 2020, en que se emite el 1er. estado de excepción, a la que llamamos prevención, la cual obedece a la declaratoria de emergencia sanitaria que emitiera el Ministerio de Salud Pública y comienza ejecutarse con la primera orden de operaciones emitida el 13 de marzo del 2019 y en la que partiendo de la organización de las Fuerzas y la jurisdicción para las operaciones permanentes de los CC.OO., el propósito principal fue activar los COE nacional y provincial en los Grupos de Trabajo (GT 1 y 2) y las Mesas Técnicas de Trabajo (MTT 1,2,3 y 4) y realizar operaciones de seguridad en apoyo al control migratorio en los pasos fronterizos y puntos de ingresos al país, apoyo logístico en el transporte de kit sanitarios y alimenticios y otras requeridas con orden, esta fase apenas duró 5 días y ante la emisión del estado de excepción el 17 de marzo, el centro de gravedad inicial cambió de un simple apoyo interinstitucional al Ministerio de Salud Pública y al Servicio Nacional de Control Migratorio a ser ya una "operación de respuesta a crisis" en apoyo al Secretaría Nacional de Gestión de Riesgos, lo que se concretaría al 19 de marzo con la emisión de la orden de operaciones No. 2. 


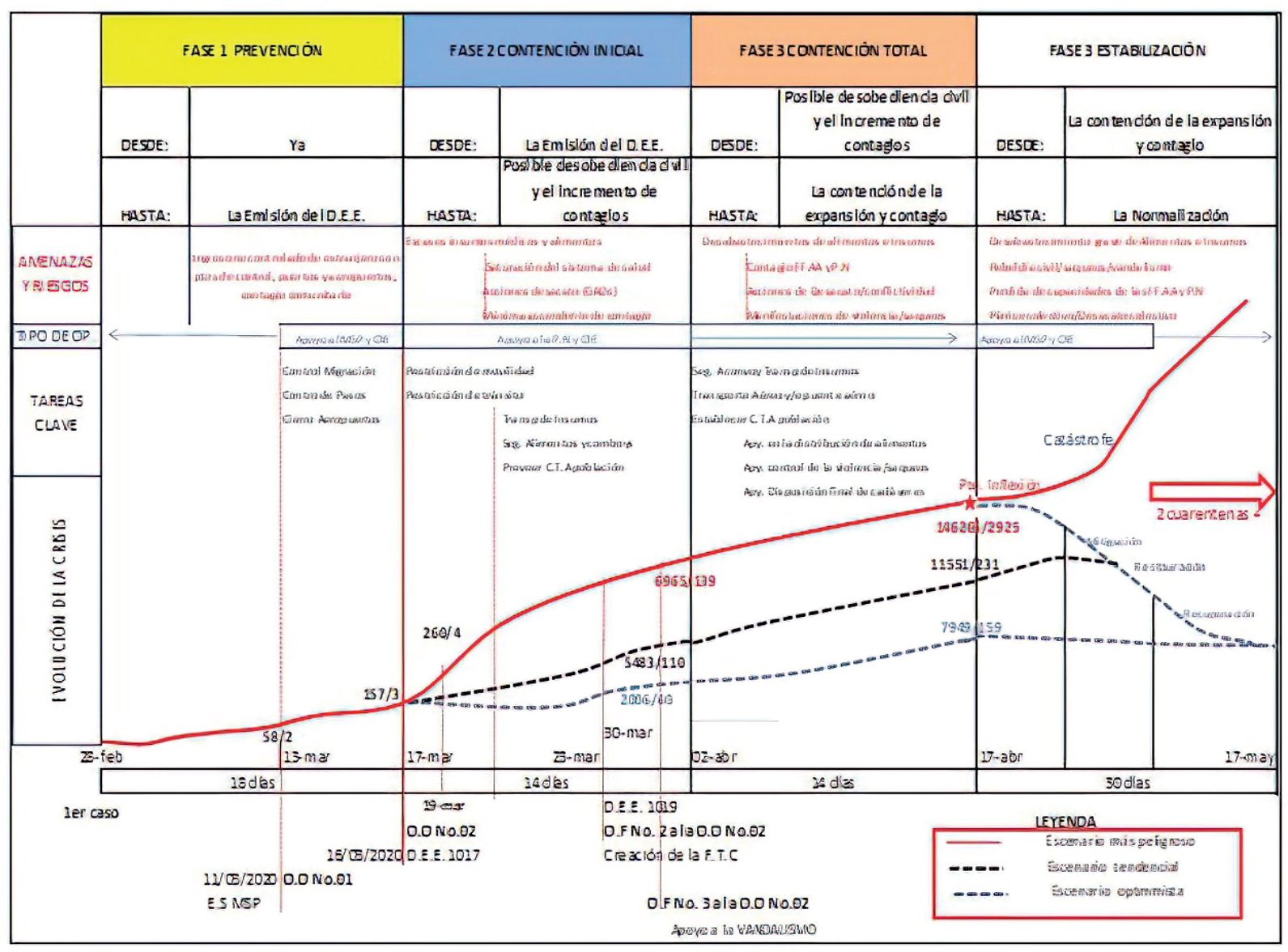

Figura 4: Modelo Operacional de la Crisis ${ }^{2}$

(2) Luego de la emisión del estado de excepción y una vez emitida la Orden de Operaciones No.2, arranca con ella la ejecución de una 2 da. fase a la que llamamos de "Contención Inicial": en que en "Respuesta a la crisis sanitaria" definida por el Comité de Operaciones de emergencia (C.O.E.) como de "calamidad pública", se enfrenta el 1er. periodo de cuarentena del 17 de marzo al 02 de abril y se configura una operación de "ámbito interno" en la que las FF.AA. irían en "apoyo a la Secretaría Nacional de Gestión de Riesgos y Emergencias (SNGRE)”, y duraría desde la emisión del ler estado de excepción hasta la "desaceleración del contagio", en donde el escenario previsible lo constituía la materialización de las siguientes amenazas y riesgos previstos:

- Escases de alimentos e insumos médicos y Saturación del sistema de salud.

- Cortes de servicios básicos.

- Acciones de desacato a las decisiones de los Gobiernos Autónomos Descentralizados que puedan desembocar en violencia social (GAD's)

- Inestabilidad en Centros de Rehabilitación

\footnotetext{
${ }^{2}$ Modificación basada en la gráfica de la pág. 44 del libro COVID 19 Cronología de una Crisis (gráfico y esquema y autoría propia).
}

\section{Social (CDR's)}

- Bloqueo de vías en protesta a las medidas tomadas

- Saqueo de convoyes

- Escases de combustible.

- Especulación de precios.

- Contagio personal de FF.AA. y P.N.

- La necesidad de Centros de Aislamiento Temporal (C.A.T) para la población.

Lo que buscamos a partir de la Orden de Operaciones No.2 y sus órdenes fragmentarias que subseguirían (4 más en total) sería minimizar la posibilidad que esas "amenazas y riesgos descritas" se materialicen y lo haríamos de allí en adelante, a través de la ejecución de tareas específicas para cada uno los momentos de la crisis que se viviría en los próximos 60 días iniciales previstos para la operación en la que el propósito principal debía ser: " la intensificación de patrullajes a lo largo del L.P.I. para apoyar el control en los pasos fronterizos autorizados e impedir el ingreso por los "pasos no autorizados" y apoyar a la Policía Nacional en la aplicación de la restricción de la movilidad de personas y vehículos, mediante el establecimiento de controles fijos integrados durante las $24 \mathrm{H}$ y patrullajes móviles durante la noche, en las principales vías de circulación, entradas y salidas de los Centros Poblados; 
en todo el territorio nacional, con especial atención a las provincias donde exista mayor densidad de contagio."

Ahora estos términos utilizados ese momento nos parecen "tan comunes", por tratar de describirlos con una sola palabra, pero si miramos en retrospectiva, a la fecha que planificábamos esta fase (el 17 de marzo) apenas 5 días después de que la OMS haya declarado "pandemia mundial" al Covid-19, fueron completamente nuevos, creados y definidos por los equipos planificadores y sus asesores, es algo que no queda escrito en ningún documento pero sucedía día a día había un nuevo concepto, una nueva tarea, una nueva misión que definir y describir.

(3) El modelo planteado preveía un posible agravamiento de la crisis, en el caso que las medidas restrictivas no fueran suficientes para controlar a la población y contener la expansión del coronavirus, éste agravamiento podía ser en todo el país o en parte de él; por ello se planificó una 3era. fase a la que le llamamos "Contención Total", inicialmente concebida para activarse en el 2 do. período de cuarentena (a partir del 02 de abril), pero debemos aceptar que nos sorprendió antes y obedeciendo a segunda hipótesis "una crisis aguda sanitaria no general sino localizada" en la provincia del Guayas; por ello ante la recomendación del CC.FF. AA. a través del Ministerio de la Defensa Nacional, el COE Nacional en sesión del 22 de marzo de 2020 crea la Zona Especial de Seguridad (Z.E.S) "Guayas", en la que se consideraba inicialmente solamente los cantones de "Guayaquil, Samborondón, Durán y Daule" sin embargo al día siguiente, el 23 de marzo de 2020; el presidente de la república emite el Decreto de Estado de Excepción 1019, cambiando la jurisdicción de la Zona Especial de Seguridad "Guayas" a "toda la provincia", por esa razón el CC.FF.AA. emite las Ordenes Fragmentarias 1 y 2 a la Orden de Operaciones No. 2 vigente a ese momento (con la que se comenzó a manejar la crisis a partir del 16 de marzo, en la cual el CC.FF.AA. reorganiza sus fuerzas y conforma la Fuerza de Tarea Conjunta "Guayas" con jurisdicción en toda esa provincia con especial atención a los cantones de "Guayaquil, Samborondón, Milagro, Durán y Daule"; lugar geográfico en donde a partir de esa fecha hasta su disolución el 15 de mayo, se estableció el "centro de gravedad" de las operaciones, activándose la III Fase de Contención Máxima, sólo para las unidades de la mencionada Fuerza de Tarea.

Es muy importante recordar que la Fuerza de Tarea Conjunta "Guayas" estuvo a cargo de tareas tan sensibles como fue el control de la población y sus recursos en los lugares donde se localizaba la "mayor taza de contagios", la ejecución del protocolo de apoyo para "la manipulación y disposición final de cadáveres" y el control de los límites provinciales de "Guayas", en 27 puntos de control interprovincial, entre los principales; ventajosamente la F.T.C., en la que se involucró la totalidad de las unidades e institutos navales, terrestres y aéreas de la jurisdicción, pudo controlar la situación y después de los 22 primeros días de operaciones la tasa de entierros diarios bajo drásticamente y la curva de contagios comenzó a descender para pronto ser sobrepasada por la provincia de Pichincha que hasta ese entonces se encontraba en el segundo lugar en velocidad de contagios y mortandad.

Para ello estableció un innovador dispositivo, que por primera vez se aplicaba en operaciones de ámbito interno, se desplazó al Comando Operacional No. 2 "Occidental" a las otras provincias de su jurisdicción natural: Manabí y Santa Elena, dividiendo la provincia del Guayas entre sus grupos operacionales, entre los que se destaca al Grupo de Tarea Conjunta "Guayaquil", conformado por las U.T. "ALFA", "BRAVO", "DELTA”, "ECHO" Y "FOXTROT", constituido por la totalidad de las unidades e institutos navales de la jurisdicción y a cargo de los cantones de Guayaquil, Samborondón, Daule y Playas, en donde se establecieron tres tipos de controles: (22) Puntos Fijos (23) Puntos móviles: y (6) Puntos críticos: conformados en sectores como mercados populares o sitios de masiva concurrencia, para que la población mantenga distancia durante su movimiento peatonal ubicadas solamente en la ciudad de Guayaquil; especial atención merece la actuación del Grupo "S" (Elemento Sanitario de Emergencia), que estuvo a cargo enteramente de la ejecución del protocolo para "la manipulación y disposición final de cadáveres"; que en ese momento fue la crisis más "mediática" nacional e internacionalmente, el Grupo "S" enterró en los meses de marzo, abril y mayo 1500 muertos diagnosticados positivos de Covid-19, en camposantos especiales destinados para el efecto.

Fue muy importante y sacrificado también el trabajo de la Fuerza de Reacción (F.R.I) del CC.FF. AA., asignada a la jurisdicción, el Grupo Especial de Comandos (G.E.K. 9) que se mantuvo en 43 días de operaciones continúas con un total de 120 efectivos, empleándose en los sectores más críticos y más contaminados de la ciudad de Guayaquil. En total hasta el cierre de sus operaciones el 15 de mayo de 2020, la FTC “Guayas” realizó 4229 operaciones, utilizando un promedio de 3016 efectivos diarios.

El 30 de marzo, ante las "alertas" emitidas por los diferentes sistemas de inteligencia, sobre la amenaza de conflictividad social, actos vandálicos, saqueos y motines en los principales Centros de Rehabilitación Social, el CC.FF.AA. se emite una nueva Orden Fragmentaria No. 3 a la Orden de Operaciones No.2 vigente, cuyo propósito principal fue el de "Apoyar a la Policía Nacional en la prevención de actos de vandalismo, violencia y saqueos", con especial atención a la Z.E.D. "Guayas", a través de: (1) La identificación en cada Zona de Defensa de los sectores comerciales y puntos de distribución de alimentos y fármacos más vulnerables ante actos de violencia, vandalismo y saqueos, (2) La intensificar los patrullajes y vigilancias en coordinación con la Policía Nacional de los centros principales de comercio de alimentos y fármacos más vulnerables, todo esto aplicando la estrategia de 
localizar "Fuerzas de Reacción", con la capacidad y las condiciones en tiempo y espacio, de neutralizar la amenaza de saqueos y la violencia cercanas a los puntos más vulnerables.

Quizá es poco conocido por la opinión pública que en cada sesión del COE Nacional se resolvía incrementar nuevas tareas y misiones adicionales a las entregadas a las FF.AA., con el propósito de contribuir en la evolución de la "detención" de la propagación del Covid-19, las mismas que se trasladaban a los Comandos Operacionales, entre las principales que merecen resaltarse, se encuentran:

1. Apoyo en la seguridad y protección de convoyes de carga pesada (productos de primera necesidad, medicamentos, bienes de consumo y productos priorizados.

2. Apoyo a la prevención y contingencia en la Zona Intangible Tagaeri y Taromenane.

3. Seguridad de los 6 corredores logísticos estratégicos de abastecimiento y producción, que garanticen la operación de transporte de carga pesada con productos de primera necesidad, para el normal flujo comercial desde: (1) Tulcán, (2) Esmeraldas y (3) Nueva Loja a Guayaquil por el norte; por el sur desde (4) Zamora (5) Huaquillas a Guayaquil y el desde (6) Alóag a Guayaquil por el centro.

4. Apoyo a la Policía Nacional ante intentos de fuga y/o amotinamiento en los Centros de Rehabilitación Social (C.R.S).

5. Seguridad de los puntos sensibles de distribución de alimentos en cada uno de los centros poblados para garantizar el abastecimiento alimenticio de la población.

6. Operaciones de protección a la infraestructura portuaria.

7. Apoyo aéreo en el transporte de carga e insumos biosanitarios.

8. Transporte humanitario nacional e internacional.

9. Transporte sanitario aéreo.

10. Puente aéreo (transporte de carga de alimentos de primera necesidad a Galápagos).

Así mismo, se operacionalizaron varios protocolos y directivas, emitidas a cada momento en las resoluciones del COE Nacional, en que resaltamos las que establecían disposiciones para los pasos fronterizos con Colombia, como el "Protocolo para el Transporte Internacional de Mercancías por Carretera por la Frontera Norte"; cuyo el esfuerzo de ejecución estuvo en responsabilidad del Comando Operacional No. 1 "Norte", el que no sólo ya venía manteniendo su esfuerzo al límite, en su tarea natural protección de fronteras norte, la importante área estratégica petrolera nororiental y minera en Imbabura, sino también incrementaba acciones de restricción de movilidad en las ciudades de su jurisdicción; por ello el aumento de estas responsabilidades significó un "esfuerzo operacional enorme" para este Comando Operacional, que sin refuerzos especiales, mantuvo la fisonomía de su frente y el control de toda su zona de defensa prácticamente sin tener incidentes de importancia o novedades especiales, hecho que casi ha pasado desapercibido en reportes e informes oficiales y prensa, pero que es necesario resaltar como un registro histórico importante.

En la concepción inicial del modelo operacional de la crisis, se preveía que "Alrededor del 17 de abril de 2020; es decir al final del 2do. Período de cuarentena, llegaríamos a un punto de inflexión en donde existirían tres posibilidades:

1. La primera que corresponde a una catástrofe en donde ante un desabastecimiento grave de alimentos e insumos, rebeldía civil/saqueos/vandalismo, pérdida de capacidades de las FF.AA. y Policía Nacional, por el masivo contagio entre sus miembros, pánico y desacato colectivo a causa de la imposibilidad de la población de acceder a recursos y sustento, hubiera pone en riesgo la supervivencia misma del Estado y sus Instituciones; si las acciones preventivas tomadas por el COE Nacional no hubiesen sido acatadas y NO se hubiesen reforzado y reducido la movilidad de la población al mínimo hasta el 17 de abril.

2. La segunda respondía a un escenario tendencial en que si bien los contagios seguían aumentando se podría a partir de la 2 da cuarentena y por el lapso de 30 días comenzar con medidas para restaurar, estabilizar y recuperar la situación de la pandemia, por lo menos en algunos sectores localizados del país, si las acciones y restricciones impuestas eran "acatadas y reforzadas por la población y las autoridades".

3. $\mathrm{Y}$ un tercer escenario optimista, en el que al 17 de abril la curva de contagios se aplanaba y estabilizaba, en donde pasaríamos directamente a la fase de estabilización para la cual sería necesario considerar un periodo adicional de un mínimo de dos cuarentenas más que serían un tiempo "clave y mínimo" en que se debe continuar con las "restricciones", para lograr los objetivos de esa fase. Ventajosamente para todos se pudo llegar al 2do escenario y esto sucedió el 15 de mayo de 2020 en que el Gobierno Nacional a través del Decreto Ejecutivo No. 1052, cambia la estrategia del Estado del Aislamiento al Distanciamiento; con lo que podríamos decir que se activa la 4ta. fase de normalización con el fin de regular la actuación de las unidades de FF.AA., en las tareas de restricción de la movilidad y direccionar el esfuerzo a la aplicación de las normas de distanciamiento social, de acuerdo a los "grados de semaforización cantonal"; así como efectivizar la "disolución de la F.TC "Guayas" y el paso de la responsabilidad al C.O 2 "Occidental", para luego en los demás CC.OO., ir entregando las responsabilidades a las instituciones y agencias en función de sus propias competencias, lo que sucedió hasta el 15 de junio del 2020, con lo cual se evidenció que nuestro modelo, coincidió casi exactamente (sólo un período más de cuarentena) con lo que sucedió en la realidad. 


\section{Diseño Operacional.}

Si bien el modelo operacional de la crisis permitió definir el plan inicial, sus fases y las tareas principales, era necesario a partir de allí completar el proceso de planificación, para encontrar en detalle entre otros aspectos: las acciones "clave" y los puntos de la "decisión" que las FF.AA, debían considerar a realizar con sus componentes (unidades especiales y comandos operacionales) para alcanzar un estado final deseado que permita alcanzar el objetivo estratégico del apoyo militar, para lograrlo entonces se desarrolló el esquema del diseño operacional para este fenómeno, en que se llegó a plantear:

El Objetivo Estratégico del Apoyo Militar, a alcanzar sería "Apoyar a las instituciones y neutralizar el riesgo de contagio", al alcanzarlo llegaríamos al Estado Final Deseado Militar, el cual se materializaría con las "instituciones del estado (SNGRE, PP.NN, MSP, entre otras) apoyadas y la pandemia controlada", dos aspectos relevantes en la estrategia a los que se llega una vez dominado el "centro de gravedad operacional" que se situaba en "frenar la transmisión de los contagios" y que dada la naturaleza multidimensional de este problema no se alcanza solos, sino con el esfuerzo coordinado de todas la demás instituciones del Estado.

El diseño operacional planteado termina definiendo 3 FASES en la operación que se pueden ir resolviendo secuencialmente a través de 16 puntos decisivos, para alcanzar un "estado final deseado" que inicialmente fue:
“Al final de las operaciones se habrá contribuido al control de la crisis, la normalización de las actividades y el bienestar de la población; manteniéndose a las FF.AA., en condiciones de continuar cumpliendo con su misión constitucional."

El desarrollo real del fenómeno pandémico, en el período que describe el presente artículo es decir desde su inicio el 12 de marzo al 15 de mayo del 2020; llegó avanzar hasta el inicio de la III FASE del Plan de Contingencia, es decir desde "la posible desobediencia civil $\mathrm{y} / \mathrm{o}$ el incremento de contagios" hasta la "contención de la expansión del contagio"; esta fase duró hasta el final de la emergencia lo que permitió ir "desacelerando" el esfuerzo operacional, a medida que se va incrementando la presencia de las instituciones locales y policiales en los diferentes cantones donde la aceleración de los contagios se mantenía controlada.

Por lo que podría decirse que con el esfuerzo de todos los componentes del poder militar, se alcanzó parcialmente el Objetivo Estratégico Militar "Instituciones apoyadas y Riesgos de contagio neutralizados", dentro de las limitaciones que estableció el Decreto de Estado de Excepción 1052 del 15 de mayo de 2020, en que se prorrogan por el lapso de 30 días "las condiciones de restricción que se establecieron en el Estado de Excepción 1017"; y se cambia la estrategia del Estado del "Aislamiento al Distanciamiento".

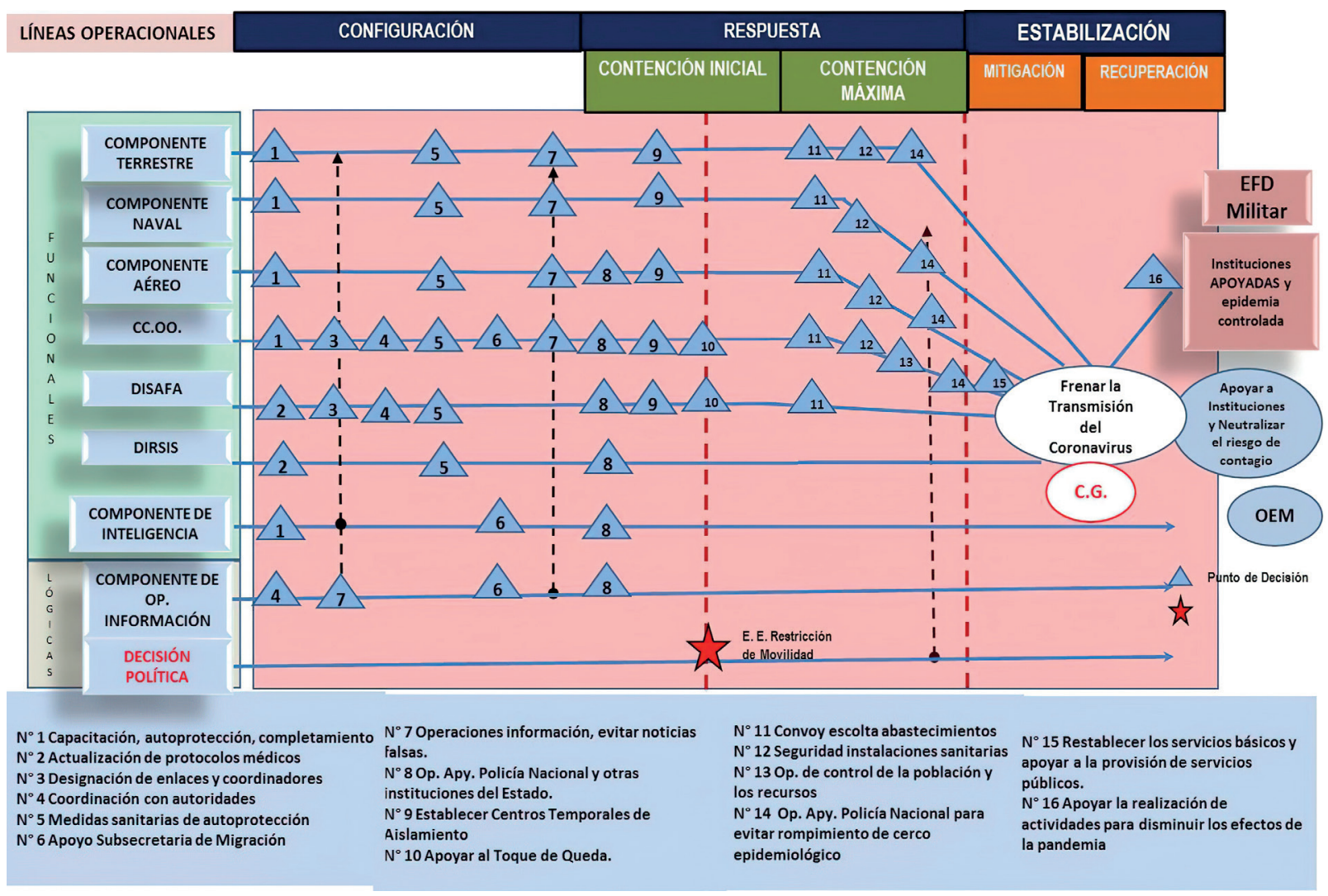

Figura 5: Diseño operacional, obtenido de Covid 19, Cronología de una crisis, pág. 37 


\section{Conclusiones}

La pandemia del COVID 19, constituye un ejemplo claro de que en las circunstancias actuales y futuras, los escenarios se encuentran plagados de incertidumbre y una diversidad de amenazas, riesgos y actores cuyas dinámicas de interrelación y manifestación, son difíciles de predecir, de igual manera, es evidente que los recursos son cada vez más escasos y están en competencia para satisfacer las demandas de la sociedad, por ello la dinámica de los escenarios, de las amenazas y del conflicto, hace difusa la línea que separa lo interno de lo externo y una visión de las amenazas por separado, estableciéndose una relación causa-consecuencia, difícil de descifrar y enfrentar, por medios lineales de deducción por lo que es necesario incorporar un pensamiento multidimensional, herramientas técnicas y elaboración de modelos para analizar una amenaza y/o fenómeno complejo, y es allí donde la prospectiva, la matemática y la informática juegan un nuevo papel vital, no solo en la comprensión del escenario operacional sino en el diseño mismo de la operación militar en el nivel estratégico, porque estas permiten establecer una relación directa entre la estrategia planteada para cumplir los objetivos y las capacidades requeridas para alcanzar ese "estado final deseado".

La crisis de la pandemia mundial del COVID-19, puso en descubierto muchas falencias en el ámbito de la gobernanza mundial para una crisis como la que se presenta, el principal problema que surgió en todos los países e instituciones fue que nadie en verdad "sabía, ni previó exactamente qué se debía hacer", parte de esa falencia se refleja en que "ningún plan militar de las FF.AA." consideró una hipótesis de amenaza como la que se presentó con la pandemia del coronavirus, es por ello que no pudimos "anclarnos de inicio a ningún plan existente", por lo que se necesitó recurrir a una planificación en crisis, que desembocó en un Plan de Contingencia y para llegar a ello fue imprescindible ahondar en el estudio del fenómeno como tal y un paso muy importante fue la creación del "modelo operacional" específico para este caso, lo que nos permitió "diseñar la operación" de manera inédita, particular y en sí misma, eso garantizó la coordinación y el aprovechamiento eficaz y eficiente de cada uno de los componentes del Comando Conjunto de las FF.AA, lo que a pesar de la "incertidumbre de la situación", permitió alcanzar en un porcentaje importante del "estado final deseado" planteado inicialmente para la operación, es decir, "las instituciones fueron apoyadas", y aunque la expansión del virus "no fue totalmente controlada", (variable que sale fuera del ámbito de las FF.AA.), debemos comprender que sucedió lo mismo sin éxito en todos los demás países de nuestro continente y la mayoría del mundo.

A través de ese complejo proceso, se llegó a establecer un Plan Matriz que se creó específicamente para apoyar a la emergencia sanitaria y la conducción de las operaciones se la realizó a través de 2 órdenes ejecutivas principales derivadas del mismo plan; en la 1era orden de operaciones el centro de gravedad se localizó en el control de los pasos fronterizos y en la 2da en el apoyo a la restricción de la movilidad, de esta 2 da. orden de operaciones se derivaron 4 órdenes fragmentarias más, las 2 primeras concernientes la creación de la F.T.C "GUAYAS", la 3era para el apoyo a la P.N en el caso de "saqueos y vandalismo" y una 4ta. y final para apoyar en el cambio de estrategia del Gobierno Nacional al pasar del "AISLAMIENTO AL DISTANCIAMIENTO" y la desactivación de la F.T.C "GUAYAS".

El máximo esfuerzo que hizo el CC.FF.AA dentro de sus competencias en apoyo a las demás instituciones del estado en especial con la Policía Nacional en el manejo de la crisis sanitaria; permitió, un proceso en que la toma de decisiones fueron inmediatas lo que permitió que el fenómeno descrito evolucione favorablemente y no se llegase al punto de inflexión del 17 de abril, donde sólo hubiesen existido 2 posibilidades, una de ellas extremadamente "negativa", que hubiese llegado amenazar la existencia misma del Estado, la cual ventajosamente con el esfuerzo de todos "no se materializó", porque se tomaron y ejecutaron las medidas de control y restricción necesarias para frenar la evolución de la expansión del virus, con el mayor esfuerzo localizado en la Provincia del Guayas en donde se manifiesto el epicentro de la crisis, pero expandido, constante y permanente a todo el país.

A la fecha de la redacción de este artículo científico, han pasado 8 meses de los acontecimientos que aquí se analizan y describen, a este tiempo no solo el Ecuador sino el mundo entero, no ha logrado controlar esta amenaza sanitaria; el "distanciamiento" es ahora, una forma de vida normal, nuevas cepas del virus y nuevas olas de contagio atacan aún a las sociedades tradicionalmente más "modernas y disciplinadas"; para nosotros esta realidad ya es parte de nuestra convivencia diaria, el trabajo cambió, el entrenamiento cambió, las operaciones se redefinieron y es posible que nada vuelva a ser como antes de marzo del 2020: pero lo que si podemos estar seguros es que "esta experiencia aprendida", debe ser escrita, debe ser estudiada, debe convertirse en doctrina, no para una pandemia similar, sino para una "contingencia" de cualquier tipo que se presente en el futuro, la forma de planificar, de simular científicamente aplicando la Ciencia y el Arte Militar será indispensable para el desempeño de los Estados Mayores enfrentados a los nuevos retos y amenazas del futuro; sólo un pensamiento multidimensional, un trabajo colaborativo y un proceso disciplinado de planificación y conducción, podrá blindarnos de alguna manera a la "incertidumbre de la evolución de las nuevas amenazas"; en parafraseo a las célebres palabras del General Eisenhower "los planes pueden no nada pero la planificación (su proceso) lo es todo". 


\section{Notas bibliográficas}

Especial agradecimiento y derechos de coautoría al equipo especial de planificación de la Dirección de Operaciones del CC.FF. AA.; bajo la dirección del G-3, mi Grab. Fabián Fuel Revelo y conformado por:

- Crnl. E.M.C. Gustavo Iturralde

- Crnl. E.M.C. Ignacio Fiallos

- Crnl. E.M.C. Xavier Riofrío

- Crnl. E.M.C. Octavio Rosales

- Crnl. E.M.C. Francisco Carpio

\section{Referencias}

Baena, P. (2010). Inteligencia Prospectiva, p.53- 62, Facultad de Ciencias Políticas y Sociales, UNAM, México.

Bartlett, H., Holman, P. \& Somes, T. (1990). Fundamentals of Force Planning (Vol. III), The Force Planning Faculty. N. W. College, Newport: Naval War College Press, USA

Cano, C. (2015). Estadistica aplicada a las Operaciones Militares, Nota de Aula Provisional, Departamento de Investigación y Doctrina, Academia de Guerra del Ejército, Ecuador.

Comando Conjunto de las FF.AA. (2020). Plan de Contingencia No. 01 de la DIGSFA a implementarse en el Sistema de Sanidad de Fuerzas Armadas ante la presencia del COVID 19, Dirección General de Sanidad del CC.FF.AA, Ecuador,

Comando Conjunto de las FF.AA. (2020) Enfrentar La Epidemia Del Coronavirus, Dirección de Operaciones del CC.FF.AA, Ecuador.

Díaz, C. (2014). Aplicación del Método de Planieamiento por Capacidades en el Ejército del Perú desde la experiencia Argentina, p.90-96, Escuela Superiror de Guerra del Ejército, Escuela de Postgrado, Perú.

Fuel, F. (2020). Covid-19 Cronología de una Crisis. Centro de Estudios Históricos del Ejército, CC.FF. AA. Ecuador.

JOINT. (2017). Chief of the Staff, Joint Planning, 5-0, Joint Doctrine Publications Hierarchy, USA.

LIPSOR. (2011). Software Mic-Mac, Laboratoire d'Investigation en Prospective Stratégie et Organisation. Francia,

Ministerio de Defensa Nacional. (s. f.). Directiva de Defensa Militar No. 01-2019-MDN, Subsecretaría de Defensa del Midena.

Ministerio de Defensa Nacional. (2020). Directiva MDN-DSS-2020-001 para "Implementar Lineamientos para contrarrestar posibles brotes de CORONAVIRUS”. Subsecretaría de Defensa del Ministerio de Defensa Nacional,. Quito.

Ministerio de Defensa Nacional. (2020). Directiva Nro. MDN-SUF-2020-002 para "Impartir disposiciones al CC.FF.AA, para el apoyo de las FF.AA a la Policía Nacional en el control de la restricción de la movilidad en el territorio nacional durante la emergencia del CORONAVIRUS", Subsecretaría de Defensa del Ministerio de Defensa Nacional. Quito.

Ministerio de Defensa Nacional Chile. (2018). Manual de Procesos de la Planificación Basada en Capacidades para la Defensa Nacional, Ministerio de Defensa Nacional. Chile.

Ministerio de Defensa Nacional, Ecuador, (2019)

Riofrío, X. (2016). El Arte Operacional. Nota de Aula Provisional, p 10-14, Departamento de Investigación y Doctrina, Academia de Guerra del Ejército, Ecuador. 